\section{A subtle case of hydroxychloroquine retinopathy: spectral domain optical coherence tomography findings}

Eye (2014) 28, 1528; doi:10.1038/eye.2014.257

Correction to: Eye (2014) 28, 1521-1522; doi:10.1038/eye.2014.212; published online 12 September 2014

Since the online publication of the above article, it has been noted that the name of the second
AC Barnes, KV Bhavsar, ML Weber and AJ Witkin

author was published incorrectly. The correct name is given above. This error has now been rectified, and the corrected article appears in this issue. The html and online pdf versions have also been rectified, and now carry the corrected paper.

The authors would like to apologise for this error. 